Ciencia y Educación, Vol. 5, No. 1, enero-abril, 2021

ISSN (impreso): 2613-8794・ISSN (en línea): 2613-8808

DOI: https://doi.org/10.22206/cyed.2021.v5i1.pp41-53

\title{
Trabajo grupal y logro académico en un ambiente virtual de aprendizaje ${ }^{\mathrm{b}}$

\author{
Collaborative Work and Academic Achievement in a \\ Virtual Learning Environment
}

\author{
Maria Carolina Moreno-Salamanca ${ }^{a}$ ORCID: 0000-0002-9130-678X
}

Recibido: 17/04/2020 • Aprobado: 4/07/2020

\begin{abstract}
Cómo citar: Moreno-Salamanca, M. C. (2021). Trabajo grupal y logro académico en un ambiente virtual de aprendizaje. Ciencia y Educación, 5(1), 41-53. https://doi.org/10.22206/cyed.2021.v5i1.pp41-53
\end{abstract}

\section{Resumen}

El presente artículo contiene los resultados de un estudio de caso, que caracteriza el trabajo de un grupo de estudiantes con logros académicos sobresalientes de la unidad de estudio de geopolítica, correspondiente a un programa de pregrado en modalidad virtual, de una universidad privada localizada en Bogotá, Colombia. El objetivo del estudio fue documentar minuciosamente las formas de trabajo, que permitieran explicar sus altos niveles de desempeño y así establecer los criterios que favorezcan la integración de grupos futuros en ambientes virtuales de aprendizaje. Los criterios de selección del grupo observado fueron los excelentes resultados académicos que obtuvieron y sus altos niveles de motivación frente al grupo. La metodología utilizada fue cualitativa; se realizaron entrevistas individuales semi-estructuradas a cada uno de los integrantes y un grupo focal. Dentro de los principales hallazgos del estudio se encuentran las formas de organización del grupo y las estrategias de trabajo colaborativo.

Palabras clave: educación; aprendizaje; educación virtual; ambiente virtual de aprendizaje; aprendizaje cooperativo; interacción.

\begin{abstract}
The present article shows the results of a case study that characterizes the collaborative work of a group of students with high academic performance in the study unit of Geopolitics in an undergraduate level program in the virtual modality, of a private university, located in Bogota, Colombia. The objective of the study was to meticulously document, the working styles of the students that allowed to comprehend their high achievement, and establish criteria that favors the integration of future groups in virtual learning environments. The selection criteria of the observed group was their high academic achievement and their outstanding motivational levels, toward the group. The used methodology was qualitative; individual interviews were conducted, in a semi-structured format to each one of the members and to a focal group. The main findings of the study include how the group built up their own strategies to collaborative processes, and their organization forms.
\end{abstract}

Keywords: education; learning; virtual education; virtual learning environment; cooperative learning; interaction.

\footnotetext{
a Universidad Pedagógica Nacional, Colombia. Correo-e: mcmorenos@upn.edu.co

b El presente artículo presenta un avance del proyecto de tesis doctoral del autor: "Análisis del Impacto de un Modelo de Aprendizaje Cooperativo en Ambientes Virtuales sobre el Rendimiento Académico de los Estudiantes Universitarios.”
} 


\section{Introducción}

La virtualidad en educación es definida por Benítez-Saza, Bustos y Arévalo (2018) como la integración de recursos educativos por medio de la internet. Este proceso hace uso de las tecnologías de la información y la comunicación (TIC), entendidas como herramientas de comunicación que apoyan el proceso pedagógico del estudiante y, por lo tanto, contribuyen a su aprendizaje (Mora y Bejarano, 2016).

La educación virtual se podría analizar de acuerdo con las oportunidades que ofrece y las debilidades que hay que superar. Con respecto a esas debilidades, Hederich-Martínez (2017) plantea tres grandes problemas: el primero, los altos niveles de deserción; el segundo, los bajos niveles de calidad, en términos del logro entre los estudiantes; y el tercero, los altos costos que limitan el acceso.

Entre las oportunidades o aspectos que se podrían aprovechar se encuentran su carácter universal, la superación de limitaciones de tiempo y espacio por los dispositivos que se usan, su fácil acceso al material multimedia; y el hecho de que este tipo de educación propicia relaciones más horizontales entre profesores y estudiantes (Hederich-Martínez, 2017). Al respecto, López y Hederich-Martínez (2010) plantean que los ambientes de aprendizaje mediados por tecnologías tienen algunas fortalezas en comparación con la enseñanza tradicional, entre las que se pueden mencionar: su potencial para que los estudiantes aprendan a su propio ritmo respetando sus diferencias individuales; su capacidad de articular diferentes formatos en forma simultánea para la presentación de la información y su capacidad para permitir modos de interacción diferenciados durante el aprendizaje, tanto individualmente, como en colaboración entre pares (Lara, Gutiérrez y Rizo, 2017; Noguera, Guerrero-Roldán y Masó, 2017; Vygotsky, 1978).

En este sentido, de acuerdo con Vega, Vidal y García (2013) el trabajo cooperativo es una propuesta pedagógica alternativa a los ambientes tradicionales.
Este tipo de trabajo puede contribuir a superar el aislamiento de los estudiantes y sus escasos niveles de motivación frente a la actividad académica, tal y como ha sido observado en ambientes presenciales de enseñanza (Järvelä et al., 2016; Johnson y Johnson, 1999; Johnson y Johnson, 2014; Johnson, Johnson y Holubec, 1994; Johnson, Johnson y Smith, 2007; Vargas y Gamboa, 2011).

Por tanto, el aprendizaje cooperativo y el trabajo colaborativo son dos herramientas didácticas para la organización de grupos educativos. Según los planteamientos teóricos de Deutsch (1949), las relaciones sociales de los estudiantes se caracterizan por ser de tres tipos: la cooperativa, la competitiva y la individual. El tipo cooperativo es definido por Deutsch (1949) como una situación y estrategia social que les permite a los estudiantes organizar sus objetivos académicos y generar relaciones positivas con sus pares para lograr un fin común. El trabajo grupal ayuda a alcanzar objetivos compartidos para maximizar el aprendizaje propio y del grupo con base en los siguientes elementos: la interacción cara a cara, la interdependencia positiva, la responsabilidad individual, las habilidades colaborativas y el procesamiento grupal (Aramendi, et al., 2014; Barkley, Croos y Major, 2007; Cardona, 2019; Hernando, Aguaded y Tirado, 2011; Herrada y Baños, 2018; Huang, et al., 2012; Kövecses-Gősi, 2018).

\section{Método}

El objetivo de este estudio se orientó a documentar minuciosamente las formas de trabajo grupal que permitieran explicar altos niveles de desempeño y así establecer los criterios que favorezcan la integración de grupos futuros en ambientes virtuales de aprendizaje. Para el desarrollo de este, se tomó como punto de partida la siguiente pregunta de investigación: ¿qué condiciones son necesarias para que un grupo de estudiantes en ambientes virtuales de aprendizaje tenga buenos resultados académicos implementando trabajo colaborativo? 
Dado el escenario planteado anteriormente, en el que se quería observar la dinámica grupal sin interferencias externas, se implementó la metodología de estudio de caso (ex post-facto ${ }^{a}$ ) con base en la investigación cualitativa que, en palabras de Hernández, Fernández y Baptista (2010) implica "comprender los fenómenos, explorándolos desde la perspectiva de los participantes en un ambiente natural y en relación con su contexto" (p. 358). En la investigación también se tuvo en cuenta las interacciones, los procesos y los logros de una situación de trabajo grupal colaborativo en un ambiente virtual de aprendizaje, a fin de vislumbrar cuáles serían los elementos del trabajo grupal que ayudarían a evitar el bajo rendimiento académico de los estudiantes.

El caso específico tuvo como foco de atención un grupo de cuatro estudiantes que solo interactúan en la plataforma educativa y nunca se han conocido en otros contextos y, a pesar de ello, logran altos desempeños académicos (Velandia, 2017). La expectativa del estudio de caso es poder detallar cómo la situación de trabajo en grupo puede dar pistas acerca de formas de interacción social (Vygotsky, 1978) verdaderamente efectivas, para ser compartidas y socializadas con futuros grupos de estudiantes en ambientes virtuales de aprendizaje.

En este análisis de caso se presentan los resultados de diferentes entrevistas y de un grupo focal realizados con estudiantes de pregrado. Se exponen los factores, las dificultades y las complejidades que tuvo el grupo para realizar el trabajo grupal.

\section{Contexto}

El contexto en el que se llevó a cabo el estudio fue en un curso de pregrado de modalidad virtual, en una universidad privada localizada en la ciudad de Bogotá. El mismo se desarrolló mediante la plataforma Learning
Management System (LMS) - Blackboard Learn.

El caso de análisis se toma del desarrollo del curso de geopolítica, incluido en un programa de pregrado, que tuvo lugar en el segundo semestre de 2019. Este, contó con la participación total de treinta y tres (33) estudiantes, donde se configuraron nueve grupos de trabajo colaborativo para el desarrollo de las actividades.

El desarrollo del curso inició con una inducción sincrónica de dos horas en la primera semana, continúa con ocho tutorías sincrónicas de dos horas de manera semanal. Se hicieron también, un encuentro virtual sincrónico de ocho horas, en la semana cinco, y un encuentro presencial o en línea de ocho horas, en la semana diez. Ninguna de las actividades anteriores era obligatoria, ya que el estudiante en el ambiente virtual de aprendizaje encuentra las grabaciones de estas actividades sincrónicas. El total de semanas trabajadas en el curso por parte de los estudiantes fue de 10 .

Las actividades que los estudiantes debían presentar durante el desarrollo del curso, para dar cuenta de su progreso académico eran: 1) un foro de presentación personal, 2) tres guías de trabajo colaborativo y autónomo que contenían las actividades y tareas específicas de la disciplina de la geopolítica internacional, 3) un foro temático asincrónico, 4) una prueba objetiva (tipo ICFES ${ }^{b}$ ) y 5) la realización de una prueba extemporánea o participación en el encuentro final (simulacro de negociación comercial internacional).

\section{El caso}

Para la identificación del caso, se examinaron al finalizar el curso los resultados académicos de la totalidad de los estudiantes del curso de geopolítica (33 estudiantes), de los cuales, el 60,6\% eran mujeres y el 39,4\% eran hombres; el rango de edad de la población era entre los 20 a 50 ańos.

a. Estud.io retrospectivo o que tiene un desarrollo anterior.

b Inst.ituto Colombiano para la Evaluación de la Educación (ICFES) 
El indicador de logro académico del curso era una valoración cuantitativa de 0 a 100 puntos posibles. Para aprobar el curso, los estudiantes debían obtener un mínimo de 60 puntos. En promedio, los resultados del curso de geopolítica fueron una media de 85.7 puntos (DS=10.2).

En el desarrollo del curso, el tutor pedía a los estudiantes que organizaran los grupos de trabajo colaborativo con un máximo de cuatro (4) estudiantes y un mínimo de dos (2). Los estudiantes, a través de la plataforma $L M S$, tienen un espacio que se denomina foro de presentación y organización de grupos, medio por el cual el grupo observado (ex post-facto) fue contactado e iniciado el proceso formativo.

Durante el curso de geopolítica se tuvieron en cuenta variables tales como: interacción, trabajo sincrónico, valoración de las actividades y mejor desempeño académico; para seleccionar el grupo de estudiantes que se iba a analizar en el desarrollo de esta investigación, posterior a la terminación del curso virtual. En este sentido, se escogió un grupo con el mejor desempeño académico, según el proceso de desarrollo de las actividades de formación y las valoraciones cuantitativas del grupo. El grupo observado estaba conformado por cuatro (4) estudiantes que serán nombrados por las siguientes letras J, K, M y N, para el análisis de esta investigación.

\section{Instrumentos}

La plataforma $L M S$ que administra los contenidos de aprendizaje tiene una herramienta llamada Blackboard Collaborate, por medio de la cual se realizaron y grabaron las entrevistas con cada uno de los estudiantes, así como el grupo focal, adelantado con todo el grupo al mismo tiempo.

Las entrevistas semiestructuradas tenían 18 preguntas; las primeras 8 preguntas indagaban por los datos personales del estudiante y las 10 preguntas siguientes versaban sobre el trabajo grupal, como se presenta en la tabla 1. El grupo focal fue orientado a través de 6 preguntas alrededor de las debilidades, las fortalezas y las dinámicas del grupo para desarrollar el trabajo en el curso, como se presenta en la tabla 2.
Tabla 1

Preguntas de la entrevista

\begin{tabular}{|c|c|}
\hline Número & Pregunta \\
\hline 1 & $\begin{array}{c}\text { ¿Cómo se sintió en la Unidad de Estudios de } \\
\text { Geopolítica y Negociación Intercultural? }\end{array}$ \\
\hline 2 & $\begin{array}{l}\text { ¿Cómo fueron los primeros momentos del } \\
\text { grupo estudio? }\end{array}$ \\
\hline 3 & $\begin{array}{c}\text { ¿Cómo empezaron la organización de las } \\
\text { actividades de la unidad de estudio? }\end{array}$ \\
\hline 4 & $\begin{array}{l}\text { Relate una sesión de trabajo normal entre el } \\
\text { grupo de trabajo }\end{array}$ \\
\hline 5 & ¿Qué opinión tiene de su grupo de trabajo? \\
\hline 6 & $\begin{array}{c}\text { ¿Cuáles fueron los medios de comunicación } \\
\text { utilizados? }\end{array}$ \\
\hline 7 & $\begin{array}{c}\text { ¿Podría hacer una valoración o hacer una } \\
\text { opinión de cada uno de los miembros del } \\
\text { grupo? }\end{array}$ \\
\hline 8 & $\begin{array}{c}\text { ¿Cuáles son las principales habilidades y } \\
\text { debilidades qué tenía cada miembro del } \\
\text { grupo? }\end{array}$ \\
\hline 9 & $\begin{array}{l}\text { ¿Hubiera preferido realizar las actividades de } \\
\text { esta unidad de estudio solo? }\end{array}$ \\
\hline 10 & $\begin{array}{c}\text { ¿Cuál es su autoevaluación en el trabajo } \\
\text { grupal? }\end{array}$ \\
\hline
\end{tabular}

\section{Tabla 2}

Preguntas del grupo focal

\begin{tabular}{|c|c|}
\hline Número & Pregunta \\
\hline 1 & $\begin{array}{c}\text { ¿Cuál cree fue el principal factor que ayudo al } \\
\text { grupo en el desempeño académico? }\end{array}$ \\
\hline 2 & ¿Algún miembro del grupo actuó como líder? \\
\hline 3 & $\begin{array}{l}\text { ¿Cómo se propuso el cronograma para la } \\
\text { realización de las actividades grupales? }\end{array}$ \\
\hline 4 & $\begin{array}{c}\text { ¿Qué herramientas tecnológicas usaron con } \\
\text { mayor frecuencia? }\end{array}$ \\
\hline 5 & $\begin{array}{c}\text { ¿Qué modificaría o qué añadiría al trabajo } \\
\text { grupal? }\end{array}$ \\
\hline 6 & $\begin{array}{c}\text { ¿Creen que es importante el trabajo en equipo } \\
\text { para el desarrollo profesional? }\end{array}$ \\
\hline
\end{tabular}

Al grupo seleccionado se le solicitó dar el consentimiento informado. Luego, se realizaron las entrevistas 
individuales, las cuales, tuvieron una duración aproximada de 20 minutos cada una. La grabación de todas las entrevistas tomó 1 hora y 34 minutos. Por otro lado, el grupo focal tuvo una duración de 31 minutos y contó con la participación de cuatro (4) estudiantes. Ambos procesos se grabaron en la plataforma Blackboard Collaborate Ultra.

\section{Procedimiento}

Lo primero que se realizó fue la gestión del consentimiento informado por cada miembro del grupo. Posteriormente, se agendaron las citas para las entrevistas y el grupo focal. Una vez realizadas, se procedió a la transcripción de ambos procesos para identificar en las grabaciones las categorías de análisis que se habían establecido a priori (interacción, comunicación, tecnologías usadas, organización del cronograma y estrategias). Se construyó una matriz de observación para desarrollar cada categoría del análisis y, finalmente, se elaboró la caracterización con los resultados del caso con base en las categorías establecidas: la interacción social del grupo (Vygotsky, 1978), tipo de comunicación, medios y tecnologías utilizadas, organización de cronograma y estrategias de trabajo colaborativo. Partiendo de esta base se hizo el análisis cualitativo de los datos.

\section{Resultados}

Los resultados del estudio de caso (ex post-facto) y los datos cualitativos que se presentan a continuación, se enmarcan en las preguntas presentadas en las tablas 1 y 2 (entrevista semi estructurada y grupo focal) y con base a las categorías de análisis establecidas para el desarrollo de la investigación.

El grupo seleccionado para el análisis posterior a la terminación del curso estuvo conformado por cuatro estudiantes tres mujeres y un hombre, con edades entre los 23 y los 48 ańos ( $\mathrm{MD}=34.5$ y $\mathrm{SD}=10.6)$, y cuyos nombres se denominarán mediante las letras mayúsculas J, K, M y N. Tres de los integrantes tienen un trabajo remunerado y el otro presta un servicio social no remunerado. Dos de ellos dijeron tener hijos.
Los estudiantes cursaban entre quinto y décimo semestre del programa de pregrado de administración de empresas. Estos estudiantes, no se conocían entre sí de forma previa y se encontraban ubicados en zonas horarias y geográficas diferentes. Este grupo obtuvo resultados excepcionalmente altos, que se ubican entre un mínimo de $98.7(\mathrm{M})$ y un máximo de 100 puntos (J, K y N).

\subsection{Interacción del grupo}

El grupo inicia su interacción mediante los dos escenarios previstos en la plataforma; el foro de presentación y la herramienta de mensajería interna. En el foro de presentación personal, los estudiantes hacen una breve biografía y plantean sus competencias y habilidades personales para iniciar la búsqueda de un grupo de trabajo. En la herramienta "mensajería interna” los estudiantes de manera asincrónica escriben un mensaje a sus compañeros para organizar y conformar los grupos de trabajo.

La primera persona en usar los dos medios de comunicación planteados para integrar grupos de trabajo en el aula fue K. Realizó su presentación personal en el foro donde describió a sí misma como: “... una persona bastante responsable, dedicada, disciplinada, amistosa, confiable y respetuosa, tranquila, positiva, alegre, me gusta tener un canal de comunicación abierto a nivel personal, profesional y familiar. Todo en el ámbito del respeto y la honestidad [...]". Luego escribió en "mensajería interna" a todo el curso "buscando un buen grupo de trabajo para la unidad de estudio". Seguidamente, en este orden, le responden J, My finalmente N. Cada uno le envía un mensaje al aula. Posteriormente, $\mathrm{K}$ conforma el grupo y envía un mensaje grupal, donde pone a los integrantes del grupo en contacto.

El inicio del trabajo en el grupo fue un momento muy importante para la integración posterior. Algunos participantes señalaron que el inicio no fue del todo fácil: “...al principio me sentí un poco perseguida, porque era un tema que no manejaba y luego ya me empecé a meter en el cuento..." ( $\mathrm{N}$, entrevista personal). Algo similar señala $\mathrm{K}$ en la entrevista: "Siempre es difícil, 
todos trabajamos en diferentes ámbitos y tenemos diferentes edades y es complicado por ese lado, como para todo grupo". Y continúa: "Al principio un poco difícil porque no nos conocíamos, no sabíamos cómo trabajaba cada uno" (K, entrevista personal).

De acuerdo con los integrantes, se hicieron diversos intentos iniciales en la búsqueda de formas efectivas de comunicación diferentes a las planteados por la plataforma LMS, por la necesidad de tener una comunicación más eficaz y ajustada a las necesidades. En particular, se veía la dificultad de que las herramientas disponibles no permiten sincronicidad en la comunicación.

\subsection{Herramientas tecnológicas para la comuni- cación del grupo}

Lo primero que intentaron en términos comunicativos fue el uso de Skype y la conformación de un grupo en la red social WhatsApp (WA), pero estas herramientas mostraron sus limitaciones. Según J, se intentó utilizar Skype en algún momento, por temas de sus viajes y conexiones, pero no fue lo suficientemente eficiente. Para $\mathrm{K}$ "[...] inicialmente formamos un grupo en $W A$, pensando que era la forma más rápida para hablar, pero nos dimos cuenta que no" $(\mathrm{K}$, entrevista). Al preguntarles el por qué no fue efectiva esta forma de comunicación, las respuestas indicaron que "WA es una red social y no una herramienta para el trabajo grupal...no permite intercambiar archivos [...]" (K, grupo focal).

La comunicación por WA resultó tan limitada, que el grupo buscó otros medios de comunicación. Las principales herramientas para la interacción del grupo fueron las incluidas en las plataformas de Google Inc., que incluyen el correo, chat, video llamada (Hangouts), compartir pantalla y almacenamiento en la nube con trabajo simultáneo en documentos o presentaciones (Drive). Estas aplicaciones les permitieron compartir archivos e información de manera sincrónica y asincrónica, lo que resultó ser muy útil en el desarrollo del trabajo grupal.

Sobre las reuniones o sesiones sincrónicas, el grupo concertó reunirse semanalmente los martes a las
20:00 horas de Colombia, así las estudiantes con hijos podían compartir con ellos un tiempo, luego de la jornada laboral. Las reuniones se hacían a través de la herramienta de video-llamada (Hangouts), para revisar las tareas individuales y grupales y tener claros los puntos de las guías. Dado que los días miércoles eran las tutorías virtuales y sincrónicas con el tutor y sus compañeros de la unidad, la reunión de los martes les permitía preparar adecuadamente ese encuentro.

Tabla 3

Tecnologías de la información y la comunicación usadas

\begin{tabular}{|c|c|c|}
\hline $\begin{array}{l}\text { Herramienta } \\
\text { tecnológica }\end{array}$ & Frecuencia de uso & Tipo de uso \\
\hline Skype & Una vez & $\begin{array}{c}\text { Primera interacción } \\
\text { virtual }\end{array}$ \\
\hline WhatsApp & Semanalmente & $\begin{array}{l}\text { Medio de } \\
\text { recordación } \\
\text { de reuniones y } \\
\text { cronograma de } \\
\text { actividades }\end{array}$ \\
\hline $\begin{array}{l}\text { Multiplataformas } \\
\text { de Google Inc }\end{array}$ & Siempre & $\begin{array}{l}\text { Uso para reuniones } \\
\text { de la herramienta } \\
\text { (Hangouts) para la } \\
\text { video llamada - } \\
\text { reunión sincrónica y } \\
\text { para la construcción } \\
\text { de trabajos } \\
\text { colaborativos por } \\
\text { medio de (Drive) }\end{array}$ \\
\hline Filmora & Frecuentemente & $\begin{array}{l}\text { Para realizar los } \\
\text { videos y trabajos } \\
\text { solicitados en el } \\
\text { curso }\end{array}$ \\
\hline $\begin{array}{l}\text { Grabaciones } \\
\text { Screem Cast }\end{array}$ & Frecuentemente & $\begin{array}{l}\text { Para realizar la } \\
\text { grabaciones de los } \\
\text { videos del curso }\end{array}$ \\
\hline Prezi & Frecuentemente & $\begin{array}{l}\text { Para realizar la } \\
\text { presentaciones } \\
\text { para el curso } \\
\text { (exposiciones) }\end{array}$ \\
\hline Plataforma $L M S$ & Semanalmente & $\begin{array}{c}\text { Para revisión } \\
\text { de contenidos, } \\
\text { calendario académico } \\
\text { e interacción con } \\
\text { pares y tutor }\end{array}$ \\
\hline
\end{tabular}




\subsection{Organización del trabajo grupal y cola- borativo}

Uno de los acuerdos iniciales que surgieron en el grupo y que puede haber tenido gran importancia en su desempeño fue que decidieron, de manera consensuada, asistir siempre a las tutorías (sincrónicas) , en tanto era también un espacio de interacción. Según $\mathrm{M}$, “[...] eso también nos servía pues ir escuchando la tutoría, y estar presentes es muy diferente a escuchar la grabación, porque, además uno puede interactuar" (M, entrevista individual). Efectivamente, los diferentes integrantes lograron estar en la gran mayoría de las tutorías, lo que les facilitó hacer preguntas e indagaciones para el desarrollo de las actividades grupales y autónomas. De igual forma, todo el grupo logró asistir al encuentro virtual planeado para la semana cinco, que tuvo una duración de ocho horas interrumpidas únicamente por la pausa para almorzar.

Durante las entrevistas individuales se le pidió a cada uno relatar lo que ocurría antes y durante las sesiones de trabajo. Al respecto, $\mathrm{N}$ dice: "Nosotros organizábamos el día y la hora en que nos íbamos a reunir, pero previamente, tratábamos de que cada uno leyera muy bien la guía y verificara las actividades que tocaba realizar en ella, para que cuando nos reuniéramos, cada uno propusiera cómo la íbamos a trabajar, cómo íbamos a desarrollar la actividad" (N, entrevista individual).

Las reuniones tenían una activa concurrencia de todos los integrantes: "En las reuniones todos participábamos, tomábamos las decisiones ahí y al final, decíamos esto fue lo que quedó, vamos hacer esto, en este orden $\mathrm{K}$ va hacer esto, J esto, $\mathrm{M}$ esto, $\mathrm{N}$ esto, $\mathrm{y}$ poníamos una fecha de entrega antes de la entrega de la guía, entonces tenemos que tener todo listo para tal fecha” (M, Entrevista Individual).

Una de las actividades iniciales que el grupo consideró más valiosas fue el hacer explícitas las fortalezas individuales: "[...] desde un principio cada uno dijo en qué era fuerte; $\mathrm{N}$ en redacción y ella iba hacer la revisión, $\mathrm{K}$ era una persona que estaba en todo, era el 'pegamento del grupo', trabajaba como con todos en conjunto, y todos desde un principio dijimos en qué éramos fuertes y se hacían las cosas de la mejor forma" ( $M$, entrevista personal). Durante las entrevistas, el grupo focal y las charlas informales con el grupo, insistieron siempre en que lograron conocer muy bien las habilidades y competencias que cada uno tenía.

$\mathrm{Al}$ indagar sobre las destrezas y las debilidades de cada estudiante, responden: "la habilidad de $\mathrm{N}$ es que es muy estratégica; su debilidad es que ella no contaba con mucho tiempo, puesto que vive en otro país, y a veces era difícil la comunicación con ella. Para J, su mayor fortaleza es su inteligencia y su debilidad es que tiene un carácter demasiado tranquilo. Para $M$, su fortaleza es que es súper pila, ella estaba pendiente de todo [...]; su debilidad debido a su personalidad es de carácter fuerte [...]" (K, entrevista individual). Por su parte $\mathrm{M}$ responde: "sobre la fortaleza de $\mathrm{N}$ es el manejo de un segundo idioma [...]; su debilidad es el poco conocimiento de las herramientas tecnologías o computacionales. Para J, su destreza es su capacidad de liderazgo; su oportunidad de mejora es su simplicidad o sencillez para la entrega de trabajos [...]. Para $\mathrm{K}$, su fortaleza es su carisma, una persona que sabe transmitir sus ideas [...]; su oportunidad de mejora es que ella cuando llegaba el momento de entregar las actividades se aceleraba un poquito" ( $M$, entrevista individual).

\subsection{Estrategias de trabajo colaborativo del grupo}

Según el grupo, el excelente desempeño que tuvieron se debió a las fortalezas que tenían, en particular, el respeto por las diferencias entre ellos. Según K "así fuéramos de diferentes edades, nos respetábamos bastante, no se pasó nunca por encima del otro, nos escuchábamos y era válida la opinión de todos" (K, entrevista individual). Otros aspectos que influyeron, de acuerdo con sus reportes, fueron el compromiso, la dedicación, la responsabilidad, el conocer las

c. Se recuerda que estas tutorías no son obligatorias para el proceso de formación de los estudiantes. 
fortalezas, las competencias y las habilidades de cada uno. Según N: "Yo creería que, la principal fortaleza del grupo fue el compromiso. Cada uno tenía claro que era lo que quería y estábamos enfocados todos hacia la misma meta. Esto hizo que unificáramos ese querer, ese objetivo, y lo plasmáramos en cada uno de los trabajos entregados. Ya que teníamos una responsabilidad muy grande para con nosotros mismos, con nuestras familias, con la misma universidad, y pues el salir adelante" (N, grupo focal). Por su parte, J: "La fortaleza del grupo, el entendimiento cuando hacíamos las distintas actividades, la comunicación y nos entendimos muy bien para crear nuevas ideas, para trabajar y desarrollar las actividades en grupo, eso se me hizo muy importante" (J, grupo focal).

En este punto es relevante agregar la adaptabilidad comunicativa y tecnológica que logró el grupo; puesto que se establecieron acuerdos sobre tiempos de entrega, características de productos esperados, protocolos de acción, sinergias en los procesos de aprendizaje, seguimiento al calendario académico y consecución de objetivos compartidos.

Aunque se indagó sobre las principales debilidades del trabajo en grupo, las observaciones y las respuestas siempre se hicieron de manera positiva. Acerca de las opiniones del grupo se puede observar una gran afinidad entre ellos: "[...]muy buen grupo, estoy en séptimo semestre; es el mejor grupo con el que me ha tocado, porque en realidad hicimos el trabajo súper bien y cumplimos con el trabajo y las fechas para hacer las entregas finales. Nos entendimos muy bien todos" (J, entrevista personal).

Cada integrante del grupo presentó su percepción sobre los otros compañeros, enfatizando principalmente las fortalezas y competencias que tenía cada uno, reconociendo la presencia de algunas diferencias entre los integrantes, tales como la edad o el carácter; al respecto dice K: "N es la mayor del grupo, ella nos decía que era la mamá de nosotros y desempeñaba ese papel pues era la mayor [...], J el mediador, él muy caballeroso con las chicas del grupo, porque era el único hombre, $[\ldots], \mathrm{M}$, es de carácter fuerte, y se le nota," (K, entrevista Individual).
Cuando se plantea un trabajo en grupo por lo general alguno de los estudiantes toma la vocería y se convierte en el líder. Sobre la pregunta si alguno de los estudiantes actúo cómo líder en el grupo; es de notar que no hubo un líder específico, sino que en cada actividad y dependiendo del tipo de habilidades, cada uno fue tomando el papel de vocero con respecto al desarrollo de la guía de estudio. "Yo creo que uno solo no, en caso y más en el trabajo de cada guía, cada uno iba tomando un rol, como te decía en la entrevista individual en lo que era experto y en algún momento todos tomamos ese rol de líder como tomando la vocería de la actividad que había que hacer, los que íbamos haciendo la actividad éramos lo que llevábamos la bandera, yo pienso eso" (M, grupo focal). $\mathrm{Al}$ respecto $\mathrm{J}$ : "Y de acuerdo con todas, hubo un líder en su momento, en cada actividad. Cada uno aportaba sus ideas y siempre nos ayudamos mutuamente. No hubo un líder especifico, pues se repartían las tareas, cada uno siempre estuvo pendiente de todo y de todos" (J, grupo focal).

La siguiente indagación es sobre si hubieran preferido trabajar las actividades del curso solos, a lo que los cuatro responden que no. "No creo que hubiera podido hacerlas de la misma manera [...], pero creo que no, por ejemplo, la parte de uso de herramientas tecnológicas me hubiera costado y estos muchachos manejan el tema al derecho y al revés" $(\mathrm{N}$, entrevista individual). Por su parte, $\mathrm{K}$ responde que "No profe para nada, hay varias razones. Entre ellas, primero son actividades bastante extensas y segundo, porque la experiencia de aprendizaje no sería igual, yo aprendí mucho de mis compañeros, y no pienso que sola no habría sido lo mismo [...]" ( $\mathrm{K}$, entrevista individual).

Sobre qué modificaciones podría hacer al grupo, las respuestas también se enfocaron principalmente hacia la unidad de estudio y no a la posibilidad de cambiar algo del grupo de trabajo. Para N: "Me gustó mucho; a pesar de no estar presencialmente en el encuentro final o negociación comercial, puesto que eso ayuda muchísimo a ver como se maneje la realidad o el mundo de los negocios como tal" (N, grupo focal). 
En general, los integrantes del grupo manifestaron haberse sentido muy bien durante su trabajo en la unidad, si bien buena parte de ese bienestar podría ser atribuido al grupo mismo. Como lo señala J: "Mira que la verdad me sentí muy cómodo, gracias al grupo que organizamos con $\mathrm{K}, \mathrm{N}$ y $\mathrm{M}$, porque fue una ayuda mutua y nos distribuimos las cargas muy bien" ( $\mathrm{J}$, entrevista individual).

Ya que el trabajo grupal tuvo fortalezas para los integrantes, se indaga sobre cómo podría esto impactar su desempeño profesional y si trabajar con otros en importante en el mundo laboral. Al respecto, “[...] claro, profe, el trabajo en equipo es súper importante, en la entrevista individual cuando tú me preguntabas que el tema del trabajo en equipo, [...] uno al principio dice, no mejor yo lo desarrollo solo [...], pero así, trabajes solo desde tu casa en un cuarto donde no veas la luz del sol, siempre necesitas del otro, para tu desarrollo y eso es súper importante; porque de alguna forma debemos estar interconectados con las personas" (M, grupo focal). Por su parte N: "Si, pues para el desarrollo profesional, en el cargo que cualquiera de nosotros desempeñemos, siempre va a requerir la ayuda del otro, es necesario que siempre trabajemos en equipo" ( $\mathrm{N}$, grupo focal).

Finalmente, se realizó una autovaloración de cada estudiante respecto a lo que aportó al trabajo grupal, es decir, se les pide hacer una autoevaluación de su propio desempeño. K: "Mi desempeño en el grupo, yo pienso que fui un miembro bastante importante, soy muy pila, proactiva, siempre estuve ahí, preguntando, pendiente del trabajo, aportando ideas, la comunicación, del 1 al 10, sería un 10 " (K, entrevista individual). Por su parte J: "Yo traté de hacer lo mejor, me pongo un 10, siempre estuve pendiente y trabajaba con la que estuviera disponible no deje ninguna guía y estuve encima de todo para que no nos cogiera la noche" (J, entrevista Individual).

\section{Discusión y conclusiones}

En el análisis de caso (ex post-facto) de esta investigación se pretendía documentar minuciosamente las formas de trabajo que permitieran explicar los altos niveles de desempeño del grupo conformado por $\mathrm{K}$, J, M y N. Además, establecer los criterios del grupo para su proceso de interacción, uso adecuado de herramientas tecnológicas y comunicativas, la organización y estrategias utilizadas en el trabajo colaborativo para obtener un excelente desempeño académico.

El grupo que conformaron $\mathrm{K}, \mathrm{J}, \mathrm{M}$ y $\mathrm{N}$ es un caso excepcional, no solo por sus logros en términos de los resultados académicos, sino por la capacidad de organización que consiguieron. La instrucción que dio el profesor en la inducción, fue de organizar un grupo de trabajo para el desarrollo del curso. Este grupo no se conocía previamente, estaban ubicados en zonas geográficas con horarias diferentes, lo anterior, no fue una dificultad para los estudiantes, sino una oportunidad de buscar las mejores herramientas de comunicación y acondicionarlas a las necesidades, lo que les permitió estar interconectados y establecer un clima de apoyo e intercambio.

Desde la conformación del grupo se generó una interacción recíproca y constante, aspecto que se refleja en las repuestas en el foro de presentación y los mensajes por medio del botón de mensajería del aula. En este sentido, la interacción es una acción de interdependencia positiva del grupo (Johnson y Johnson, 1999), lo que contribuyó directamente en el mejoramiento de las habilidades sociales y resolutivas, los procesos metacognitivos, las capacidades para trabajar en grupo, para aprender y negociar entre ellos (Bosch, Mentz y Reitsma, 2019).

El grupo presentó una interrelación permanente por medio de las diferentes herramientas de comunicación del aula virtual, y otras, que se fueron acomodando para el desarrollo del trabajo durante curso. Sobre este punto, la exploración del grupo para hallar el mejor medio para la interacción sincrónica se ve reflejado en el uso de diferentes herramientas de tecnología y comunicación, las que fueron ensayando para poder llegar a establecer el medio más idóneo de interacción en las reuniones semanales que realizaban. Por tanto, las herramientas de las plataformas de Google facilitaron trabajar sincrónicamente en el desarrollo de las 
actividades y tareas del curso, de una manera adecuada y eficiente. Este aspecto, presenta la valiosa necesidad del trabajo grupal de realizar encuentros cara a cara sincrónicos- (Cañabate et. al., 2019; Cardona, 2019), lo que les permitió a los estudiantes mejorar la relación entre ellos, generar adaptación y conocer las competencias sociales de sus compañeros.

En este análisis del caso, se muestra la importancia de la organización de pequeños grupos en ambientes virtuales para el desarrollo del trabajo colaborativo, lo que se evidencia con claridad al interior del equipo; pues cada participante tenía claro su rol, sus responsabilidades individuales y la interdependencia entre los miembros para establecer un cronograma adecuado de trabajo, horarios, fechas y objetivos compartidos, con el fin de aprender y obtener el mejor resultado académico posible.

Lo anterior, se ve reflejado en las estrategias de trabajo colaborativo que el grupo usó para lograr los objetivos, entre las que se encuentran el conocer las habilidades y competencias de cada uno de los miembros del grupo. Un ejemplo claro es el hecho que $\mathrm{N}$ era la que tenía la mejor competencia en uso del inglés, por vivir fuera del país. Por otro lado, J tenía notorias cualidades de liderazgo, además habilidades en el uso de herramientas tecnológicas.

El grupo se organizó también pensado en el hecho que $\mathrm{N}$ y $\mathrm{M}$ tienen hijos, por tanto, la necesidad de que los horarios y fechas para trabajar no coincidieran con los tiempos en familia, un aspecto significativo para mediar las necesidades e intereses de cada miembro del grupo. Asimismo, los integrantes se tomaron el trabajo de conocer los estilos de aprendizaje y habilidades de cada uno; quién es el mejor para redactar, para buscar información, para organizar las actividades, y así, cada vez se generaba un mejor conocimiento de las capacidades de cada miembro del grupo, lo que los lleva a obtener los mejores logros académicos y una sinergia grupal para la presentación de las tareas y actividades.

Los hallazgos de la investigación muestran la excelente coordinación, integración, conocimiento de cada miembro del grupo, un manejo claro y adecuado con el material, los conocimientos y el cronograma del curso. Así mismo, la cohesión, el trabajo colaborativo y cooperativo del grupo, les permitió llegar a las metas y objetivos colectivos propuestos (Saekhowa, 2015) para el aprendizaje de la geopolítica.

Por otro lado, el grupo generó motivación, regulación, interacción social (Vygotsky, 1978), organización metodológica (un cronograma propio de trabajo, gestión y organizar tiempos), habilidades sociales y de comunicación; permitiendo un buen desarrollo y el desempeño del grupo para alcanzar sinergia, dinámica, gran disposición y deseo de trabajar en grupo para aprender entre ellos. Lo expuesto, se vio reflejado en sus excelentes valoraciones y los buenos resultados académicos.

El estudio permitió establecer la importancia de la organización de grupos de trabajo en entornos virtuales de aprendizaje y el desarrollo de habilidades sociales de los estudiantes. A pesar de lo anterior, este curso no estuvo estructurado bajo un modelo de aprendizaje cooperativo en sentido estricto. El grupo no se integró bajo esos conceptos específicos, sin embargo, sí se logran algunos elementos característicos de este tipo de aprendizaje. Un ejemplo de lo anterior es la interdependencia social o positiva (Johnson y Johnson, 1999), la cual, se ve reflejada entre los estudiantes, quienes expresaron claramente que el hecho de darse a la tarea de conocer al otro por medio de sus habilidades, competencias y fortalezas ayudó al logro académico del grupo. La responsabilidad individual (Johnson y Johnson, 1999) fue un punto crucial para el desarrollo del trabajo grupal, ya que cada uno se propuso dar lo mejor de sí mismo y tener un alto nivel de compromiso para entregar lo que correspondía en cada una de las guías y tareas. La interacción cara a cara (Johnson y Johnson, 1999) se puede observar en los medios de comunicación utilizados por el grupo para las reuniones sincrónicas de trabajo semanal.

Como se evidenció en los resultados, una herramienta como WA no fue eficiente, pues era necesaria 
una interacción más cercana, directa y afable. Esto lo lograron por medio de las herramientas de Google, las cuales permitieron el uso de video llamadas, integradas a otros medios virtuales (correo, chat, Hangouts, compartir pantalla y almacenamiento en la nube con trabajo simultáneo en documentos o presentaciones-Drive-).

Las habilidades sociales o interpersonales (Johnson y Johnson, 1999) se reflejaron claramente en el hecho que el grupo tuvo como elemento básico de interacción el respeto, el conocer qué habilidades y competencias tenía cada uno de los participantes, además de integrarse o "complementarse en el trabajo del curso". Otro aspecto relevante del grupo, fue el tema del liderazgo, ya que no hubo un miembro único liderando el proceso, sino que dependía del tipo de actividad y trabajos a entregar, que se daba un tipo de liderazgo rotativo o por roles, atribuido a las fortalezas que cada uno tenía.

En el proceso grupal (auto monitoreo y autoeficacia), el grupo demostró su capacidad de regulación social compartida (Järvelä et al., 2016), ya que se recordaban las citas mutuamente, tenían claras las fechas y horas de las sesiones de trabajo sincrónico y, además, seguían puntualmente el calendario de las actividades propuesto en el aula, lo que les permitió dar cumplimiento con el proceso. Sobre la evaluación como proceso constante, se ve reflejada en las valoraciones finales y el desempeño general de los participantes en el desarrollo del curso.

Esta investigación evidenció la importancia que tiene el desarrollo de las habilidades personales y sociales en grupos de trabajo colaborativo, en ambientes virtuales de aprendizaje; lo que permite que los miembros de un grupo generen interacción con el conocimiento y los materiales, así como una interrelación social positiva con sus pares; aspectos que impacta evidentemente en el desempeño académico y la comunicación efectiva entre estudiantes en modalidad virtual.

También el grupo observado proporcionó elementos sobre cómo podría fluir el aprendizaje, la construcción cooperativa del conocimiento (Johnson y Johnson, 1999), la comunicación de calidad y adaptativa, la interacción significativa, la elaboración colaborativa de las tareas, la responsabilidad individual y colectiva en pro de los objetivos comunes. Sin embargo, el estudio también presentó que hay algunas aspectos a mejorar en la organización de grupos en ambientes virtuales de aprendizaje, tales como: la adaptación de los modelos educativos virtuales (pasar de la competencia e individualidad a la cooperación -Vargas y Gamboa, 2011); la labor docente debe estar enfocada en aspectos pedagógicos, didácticos, instruccionales y acompañamiento permanente a los estudiantes, para que no sienta aislamiento social en su proceso de formación; o el uso y manejo de herramientas tecnológicas adecuadas para la educación virtual y el trabajo cooperativo. Esto último, debido a que en la mayoría de los modelos pedagógicos en aulas virtuales se genera una división de las tareas de los cursos y no una co-construcción colectiva del conocimiento.

Finalmente, el estudio evidenció que la interacción social y el desarrollo de habilidades en los entornos virtuales de aprendizaje podrían disminuir el abandono escolar por la vía del apoyo social, el acompañamiento y la motivación. $\mathrm{Al}$ respecto, dice $\mathrm{M}$ :

"Te cuento mi experiencia un poco: yo estudie hace 10 años presencial en esta misma universidad, llegué hasta octavo semestre. Por temas personales no terminé, me retiré de estudiar. La universidad me llamó para que terminara la carrera en la modalidad virtual. El ańo pasado fue mi primer semestre virtual, y me di cuenta de que en esta modalidad uno solo no puede. Puesto que hay mucha información, lecturas, responsabilidades, el tiempo que hay que dedicar a cada actividad es bastante. Por eso, el trabajo cooperativo le sirve a uno mucho, porque le ayuda a entender temas, desde otro punto de vista y sirve para avanzar más rápido teniendo contacto con alguien más. Sino no fuera así, uno sola se ahoga" (M, entrevista individual). 


\section{Referencias}

Aramendi, P., Bujan, K., Garín, S. y Vega, A. (2014). Estudio de caso y aprendizaje cooperativo en la universidad. Profesorado: Revista de Curriculum y Formación del Profesorado, 18(1), 413-429. https://goo.gl/yZ79hU

Barkley, E., Croos, P. y Major, C. (2007). Técnicas de aprendizaje colaborativo. Madrid: Morata.

Benitez-Saza, C., Bustos, E. y Arévalo, E. (2018). The Society of Information and Communication in Education: Speeches Configuring the Teacher for Virtual Education: Subjective and Subjectivity. Revista Cientifica. 32(2), 183-192.

Bosch, C., Mentz, E. y Reitsma, G. (2019). Integrating Cooperative Learning into the Combined Blended Learning Design Model: Implications for Students' Intrinsic Motivation. International Journal of Mobile and Blended Learning, 11(1), 58-73.

Cañabate, D., Serra, T., Bubnys, R. y Colomer, J. (2019). Pre-Service Teachers' Reflections on Cooperative Learning: Instructional Approaches and Identity Construction. Sustainability, MDPI, Open Access Journal, 11(21). https://doi. org/10.3390/su11215970

Cardona, A. (2019). El Aprendizaje Cooperativo como Estrategia Didáctica para el Desarrollo de Habilidades en la Solución de Problemas Contextualizados con Situaciones Aditivas, Para Estudiantes De Grado $5^{\circ}$. Facultad de Humanidades y Ciencias Sociales. Maestría En Educación. Cali: Universidad Javeriana. http://hdl. handle.net/11522/11370

Deutsch, M. (1949). A theory of cooperation and competition. Human Relations. (2), 129-152.

Hederich-Martínez, C. (2017). Las Expectativas frustradas de la educación virtual: ¿Cuestión del Estilo? Educación y Tecnologías de la Información y la Comunicación. Cátedra Doctoral 3. Bogotá: Universidad Pedagógica Nacional de Colombia.
Hernández Sampieri, R., Fernández, C. y Baptista, P. (2010). Metodología de la investigación. (5ta ed.) México D.F.: The McGraw-Hill Companies, Inc.

Hernando, A., Aguaded, J. y Tirado, R. (2011). Aprendizaje cooperativo «on-line» a través del Campus Andaluz. Análisis de las interacciones. Enseñanza \& Teaching: Revista Interuniversitaria de Didáctica. 29(1), 135-158. https://cutt.ly/NjwJfhU

Herrada, R. y Baños, R. (2018). Aprendizaje cooperativo a través de las nuevas tecnologías: Una revisión. @tic Revista D'Innovació Educativa, (20), 16-25.

Huang, M., Hsiao, W., Chang, T. y Hu, M. (2012). Design and Implementation of a Cooperative Learning System for Digital Content Design Curriculum: Investigation on Learning Effectiveness and Social Presence. TOJET: The Turkish Online Journal of Educational Techno$\log y$ - October 2012, (11), 4. https://eric.ed. gov/?id=EJ989259

Järvelä, S., Kirschner, P., Hadwin, A., Järvenoja, H., Malmberg, J., Miller, M. y Laru, J. (2016). Socially shared regulation of learning in CSCL: understanding and prompting individual- and group-level shared regulatory activities. International Journal of Computer-Supported Collaborative Learning. 11(3); 263-280. https://doi. org/10.1007/s1 1412-016-9238-2

Johnson, D. y Johnson, R. (2014). Cooperative learning in 21st century. 785-811. http://www.redalyc. org/pdf/167/16731690008.pdf

Johnson, D. Johnson, R. y Smith, K. (2007). The State of Cooperative Learning in Postsecondary and Professional Settings. https://cutt.ly/5jwLizj

Johnson, D. y Johnson, R. (1999). El aprendizaje cooperativo en el aula. https://cutt.ly/ojwLWJq

Johnson, D., Johnson, R. y Holubec, E. (1994). New circles of learning: Cooperation in the classroom. Alexandria, VA: Association for Supervision \& Curriculum Development. 
Kövecses-Gősi, V. (2018). Cooperative Learning in VR Environment. Acta Polytechnica Hungarica. 15(3). https://cutt.ly/RjwLJKf

Lara, L., Gutiérrez, L. y Rizo, R. (2017). Características del Escenario Interactivo de Aprendizaje como ambiente pedagógico. Educación científica e inclusión sociodigital: actas del IX Congreso Iberoamericano de Educación Científica y del I Seminario de Inclusión Educativa y Sociodigital (CIEDUC 2017). https://cutt.ly/RjwZqCc

López, O. y Hederich-Martínez, C. (2010). Efecto de un andamiaje para facilitar el aprendizaje autorregulado en ambientes hipermedia. Revista Colombiana de Educación, (58), 14-39. https:// cutt.ly/RjwZqCc

Mora, D. y Bejarano, G. (2016). Prácticas educativas en ambientes virtuales de aprendizaje. Aletheia, 8(2), 48-63.

Noguera, I., Guerrero-Roldán, A. y Masó, R. (2017). Collaborative agile learning in online environments: Strategies for improving team regulation and project management, Computers \& Education (2017). https://cutt.ly/YjwXcHg

Saekhowa, J. (2015). Steps of Cooperative Learning on Social Networking by Integrating Instructional Design based on Constructivist Approach. Procedia - Social and Behavioral Sciences, (197), 1740-1744. https://cutt.ly/wjwXLx9
Vargas, G. y Gamboa, S. (2011). Didáctica en la Condición Postmoderna. De las Competencias a la Cooperación. Revista Digital de Investigación la Saliana (3), 20-30. https://cutt.ly/1jwCieg

Vega, L. Vidal, D. y García, M. (2013). Avances Acerca de los Efectos del Aprendizaje Cooperativo sobre el Logro Académico y las Habilidades Sociales en Relación con el Estilo Cognitivo. Revista Colombiana de Educación, (64), 155-174. Bogotá: Universidad Pedagógica Nacional. https://doi.org/10.17227/0120391 6.64rce155.174

Velandia, N. (2017). Logro Educativo Comparado Entre Educación Superior Presencial y Virtual en Colombia: Algunas Tendencias y Grandes Desafíos. https://cutt.ly/YjwCg9q

Vygotsky, L. (1978). Mind in Society: The Development of the Higher Psychological Processes. Cambridge, Massachusetts: Harvard University Press (Edited by M. Cole et al). 